\title{
STRONG SOLUTIONS TO 3D-LAGRANGIAN AVERAGED BOUSSINESQ SYSTEM
}

\author{
RIDHA SELMI ${ }^{1,3,4, *}$, LEILA AZEM ${ }^{2,4}$ \\ ${ }^{1}$ Department of Mathematics, College of Sciences, Northern Border University, P.O. Box 1321, Arar, \\ 73222, KSA \\ ${ }^{2}$ Department of Mathematics, College of Sciences and Art (TURAIF), Northern Border University, KSA \\ ${ }^{3}$ Department of Mathematics, Faculty of Sciences of Gabès, University of Gabès, Gabès, 6072, Tunisia \\ ${ }^{4}$ Laboratory of partial differential equations and applications (LR03ES04), Faculty of sciences of Tunis, \\ University of Tunis El Manar,Tunis, 1068, Tunisia \\ *Corresponding author: Ridha.selmi@nbu.edu.sa
}

\begin{abstract}
Under suitable assumptions on the initial data, we prove the existence, uniqueness of the strong solutions to a regularized periodic three-dimensional Lagrangian averaged Boussinesq system, in a Sobolev spaces. Also, we establish the convergence results of this unique strong solution of this regularized Boussinesq system to a strong solution of the three-dimensional Boussinesq system, as the regularizing parameter vanishes.
\end{abstract}

\section{INTRODUCTION AND STATEMENT OF MAIN RESULTS}

We consider the following 3D-Lagrangian averaged Boussinesq- $\alpha$ system:

Received April $4^{\text {th }}, 2020$; accepted April 22 ${ }^{\text {nd }}, 2020$; published December 17 $7^{\text {th }}, 2020$.

2010 Mathematics Subject Classification. primary 35A01, 35A02, 35B40; secondary 35B10.

Key words and phrases. 3D-Lagrangian averaged Boussinesq- $\alpha$ system; alpha-regularization; existence; uniqueness; convergence.

(C)2021 Authors retain the copyrights of their papers, and all open access articles are distributed under the terms of the Creative Commons Attribution License. 


$$
\left\{\begin{array}{l}
\frac{\partial v}{\partial t}+(u \cdot \nabla) v+\nabla u^{T} \cdot v+\nabla p=\nu \Delta v+\theta e_{3}, \quad(t, x) \in \mathbb{R}_{+} \times \mathbb{T}^{3} \\
\frac{\partial \theta}{\partial t}-\kappa \Delta \theta+(u \cdot \nabla) \theta=0, \quad(t, x) \in \mathbb{R}_{+} \times \mathbb{T}^{3} \\
v=\left(1-\alpha^{2} \Delta\right) u, \quad(t, x) \in \mathbb{R}_{+} \times \mathbb{T}^{3} \\
\operatorname{div} u=\operatorname{div} v=0, \quad(t, x) \in \mathbb{R}_{+} \times \mathbb{T}^{3} \\
(u, \theta)_{\left.\right|_{t=0}}=\left(u_{0}, \theta_{0}\right), \quad x \in \mathbb{T}^{3},
\end{array}\right.
$$

where by $\mathbb{T}^{3}$ we refer to the $3 D$-torus, $\nu>0$ represents the viscosity of the fluid and $\kappa>0$ its thermal conductivity. The unknown vector field $u$, the scalars $p$ and $\theta$ stand respectively for the velocity, the pressure and the temperature of the fluid at the point $(t, x) \in \mathbb{R}_{+} \times \mathbb{T}^{3}$. The superscript $M^{T}$ denotes the transpose of the matrix $M$. The data $\left(u_{0}, \theta_{0}\right)$, are respectively the initial free divergence velocity and the initial temperature. The Lagrangian averaged Boussinesq- $\alpha$ model (1.1) is the first to use Lagrangian averaging to address the turbulence closure problem and also in geophysical modeling [4]. The Boussinesq equations were derived in the nineteenth century by Joseph Boussinesq, despite intense study, there still remain many difficulties and open questions concerning them. Namely, the Boussinesq system have an incomplete solution theory. It is not known whether global in time strong solutions exist, and although we know weak solutions exist, we are still unable to prove their uniqueness. Regularization models are a way to come up with a rather well-posed problem theory. The first attempt to regularize the Navier-Stokes equations was made in [9] via smoothing the convective velocity by taking the convolution product against a mollifier. Here, and among many others methods of regularization presented in the literature, we note that $\alpha$-regularization models are obtained by applying a smoothing via taking the inverse of the Helmholtz operator $I-\alpha^{2} \Delta$. The interested reader is referred to [3,5,6]. Especially, the LANS- $\alpha$ model acts by modifying the nonlinearity in the Navier-Stokes equations without introducing any extra dissipation [6]. It can be seen as a systematic method for modelling the mean circulatory effects of small-scale turbulence, while preserving the mathematical properties that guarantee existence of a unique, regular solution $[3,5]$. The inviscid Lagrangian averaged Euler- $\alpha$ equations were originally derived as Euler-Poincaré equations in the framework of Hamilton's principle for geometric fluid mechanics [7]. The existence, uniqueness and continuous dependance of solutions to initial date, as well as convergence results of various $\alpha$-models, as $\alpha$ vanishes can be found in $[1,2,8,10,12]$ and references therein. Before stating our main results, let us introduce some notations that will be used throughout the paper. 
- For $n \in \mathbb{N}$, we denote by $P_{n}$ the projection into Fourier modes of order up to $n$, that is

$$
P_{n}\left(\sum_{k \in \mathbb{Z}^{3}} \widehat{u}_{k} e^{i k x}\right)=\sum_{|k| \leq n} \widehat{u}_{k} e^{i k x}
$$

- For $s>0$, we define the operator $\Lambda^{s}$ acting on $H^{s}\left(\mathbf{T}^{3}\right)$ as follows. Let $u \in H^{s}\left(\mathbb{T}^{3}\right)$ having the Fourier series

$$
u(x)=\sum_{k \in \mathbb{Z}^{3}} \widehat{u}_{k} e^{i k x} \in H^{s}\left(\mathbb{T}^{3}\right)
$$

Then, we define

$$
\Lambda^{s} u(x)=\sum_{k \in \mathbb{Z}^{3}}|k|^{s} \widehat{u}_{k} e^{i k x} \in L^{2}\left(\mathbb{T}^{3}\right) .
$$

- We denote by $\|\cdot\|_{\dot{H}^{s}}$ the seminorm $\left\|\Lambda^{s} \cdot\right\|_{L^{2}}$. This is compatible with the definition of the Sobolev norm. In fact, $\|\cdot\|_{H^{s}}$ is equivalent to $\|\cdot\|_{L^{2}}+\|\cdot\|_{\dot{H}^{s}}$. Note that in the Fourier setting it is more usual to define an equivalent norm on $H^{s}$ by

$$
\|u\|=\left(\sum_{k \in \mathbb{Z}^{3}}\left(1+|k|^{2 s}\right)\left|\widehat{u}_{k}\right|^{2}\right)^{1 / 2} .
$$

- We refer to the fractional Laplacian $\sqrt{-\Delta}$ by $\Lambda$.

- We denote

$$
\begin{gathered}
B(u, v)=[(u \cdot \nabla) v], \quad u, v \in H^{1}\left(\mathbb{T}^{3}\right), \\
\widetilde{B}(u, v)=[(\nabla \times v) \times u], \quad u, v \in H^{1}\left(\mathbb{T}^{3}\right) .
\end{gathered}
$$

- We define the space $\widetilde{H}^{s}=\left\{u \in H^{s}, \quad \operatorname{div} u=0\right\}$. We remark that, for $u, v$ and $w \in \widetilde{H}^{1}\left(\mathbb{T}^{3}\right)$

$$
(B(u, v), w)=-(B(u, w), v) .
$$

- Due to the identity

$$
(u \cdot \nabla) v+\sum_{j=1}^{3} v_{j} \nabla u_{j}=-u \times(\nabla \times v)+\nabla(v \cdot u),
$$

we obtain

$$
(\tilde{B}(u, v), w)=(B(u, v), w)-(B(w, v), u)
$$

- By the definitions of the operators $B(u, v)$ and $\widetilde{B}(u, v)$, we deduce the following properties:

Lemma 1.1. i) Let $u, v, w \in \tilde{H}^{1}\left(\mathbb{T}^{3}\right)$, then

$$
(B(u, v), w)=-(B(u, w), v)
$$

which in turn implies that

$$
(B(u, v), v)=0, \quad u, v \in \tilde{H}^{1}\left(\mathbb{T}^{3}\right) .
$$


Also,

$$
(\tilde{B}(u, v), w)=(B(u, v), w)-(B(w, v), u), \quad u, v, w \in \tilde{H}^{1}\left(\mathbb{T}^{3}\right)
$$

and hence

$$
(\tilde{B}(u, v), u)=0, \quad u, v \in \tilde{H}^{1}\left(\mathbb{T}^{3}\right)
$$

ii) Let $u \in \tilde{H}^{1}\left(\mathbb{T}^{3}\right), v \in \tilde{H}^{2}\left(\mathbb{T}^{3}\right)$, and $w \in L^{2}\left(\mathbb{T}^{3}\right)$, then

$$
|(B(u, v), w)| \leq c\|\nabla u\|_{L^{2}}\|\nabla v\|_{L^{2}}^{1 / 2}\|\triangle v\|_{L^{2}}^{1 / 2}\|w\|_{L^{2}} .
$$

iii) Let $u, v, w \in \tilde{H}^{1}\left(\mathbb{T}^{3}\right)$, then

$$
\left|\langle\tilde{B}(u, v), w\rangle_{\tilde{H}^{-1}\left(\mathbb{T}^{3}\right)}\right| \leq c\|\nabla u\|_{L^{2}}\|\nabla v\|_{L^{2}}\|w\|_{L^{2}}^{1 / 2}\|\nabla w\|_{L^{2}} .
$$

Here $<., .>$ denotes the duality pairing of $\tilde{H}^{1}\left(\mathbb{T}^{3}\right)$ and $\tilde{H}^{-1}\left(\mathbb{T}^{3}\right)$.

iv) Let $u \in \tilde{H}^{1}\left(\mathbb{T}^{3}\right), v \in L^{2}\left(\mathbb{T}^{3}\right)$ and $w \in \tilde{H}^{2}\left(\mathbb{T}^{3}\right)$, then

$$
\begin{aligned}
\left|\langle\tilde{B}(u, v), w\rangle_{H^{-2}}\right| & \leq c\left(\|u\|_{L^{2}}^{1 / 2}\|\nabla u\|_{L^{2}}^{1 / 2}\|v\|_{L^{2}}\|\Delta w\|_{L^{2}}\right. \\
& \left.+\|v\|_{L^{2}}\|\nabla u\|_{L^{2}}\|\nabla w\|_{L^{2}}^{1 / 2}\|\Delta w\|_{L^{2}}^{1 / 2}\right) .
\end{aligned}
$$

Proof. See [10] and references therein.

Using the above notations and the identity (1.2), we obtain the following equivalent systems of equations:

$$
\left\{\begin{array}{l}
\frac{\partial v}{\partial t}+\tilde{B}(u, v)-\nu \triangle v=\theta e_{3}, \quad(t, x) \in \mathbb{R}_{+} \times \mathbb{T}^{3} \\
\frac{\partial \theta}{\partial t}-\kappa \triangle \theta+B(u, \theta)=0, \quad(t, x) \in \mathbb{R}_{+} \times \mathbb{T}^{3} \\
v=\left(1-\alpha^{2} \triangle\right) u, \quad(t, x) \in \mathbb{R}_{+} \times \mathbb{T}^{3} \\
\operatorname{div} u=\operatorname{div} v=0, \quad(t, x) \in \mathbb{R}_{+} \times \mathbb{T}^{3} \\
(u, \theta)_{\left.\right|_{t=0}}=\left(u_{0}, \theta_{0}\right), \quad x \in \mathbb{T}^{3},
\end{array}\right.
$$

Our first result is the following existence and uniqueness theorem.

Theorem 1.1. Let $\theta^{0} \in \dot{H}^{1}\left(\mathbb{T}^{3}\right)$ and $u^{0} \in \dot{H}^{2}\left(\mathbb{T}^{3}\right)$ a divergence free vector field. Then, there exists a unique strong solution $\left(u_{\alpha}, \theta_{\alpha}\right)$ of system (1.10), such that

$$
u_{\alpha} \in C\left(\mathbb{R}_{+}, \dot{H}^{2}\left(\mathbb{T}^{3}\right)\right) \cap L_{\text {loc }}^{2}\left(\mathbb{R}_{+}, \dot{H}^{3}\left(\mathbb{T}^{3}\right)\right)
$$

and

$$
\theta_{\alpha} \in C\left(\mathbb{R}_{+}, \dot{H}^{1}\left(\mathbb{T}^{3}\right)\right) \cap L_{l o c}^{2}\left(\mathbb{R}_{+}, \dot{H}^{2}\left(\mathbb{T}^{3}\right)\right)
$$


Moreover, $\forall \quad 0 \leq t \leq T$, this solution satisfies the following energy estimates

$$
\begin{aligned}
\left\|\nabla \theta_{\alpha}\right\|_{L^{2}\left(\mathbb{T}^{3}\right)}^{2} & +\left\|\nabla u_{\alpha}\right\|_{L^{2}\left(\mathbb{T}^{3}\right)}^{2}+\alpha^{2}\left\|\Delta u_{\alpha}\right\|_{L^{2}\left(\mathbb{T}^{3}\right)}^{2}+\kappa \int_{0}^{t}\left\|\Delta \theta_{\alpha}\right\|_{L^{2}\left(\mathbb{T}^{3}\right)}^{2} d \tau \\
& +\nu \int_{0}^{t}\left(\left\|\Delta u_{\alpha}\right\|_{L^{2}\left(\mathbb{T}^{3}\right)}^{2}+\alpha^{2}\left\|\nabla \Delta u_{\alpha}\right\|_{L^{2}\left(\mathbb{T}^{3}\right)}^{2}\right) d \tau \leq \mathcal{K}_{\alpha}(T),
\end{aligned}
$$

where

$$
\mathcal{K}_{\alpha}(T)=\frac{K(T)}{2 \mu}+\frac{K^{3}(T)}{2 \mu \alpha^{6}}\left(1+\alpha^{2}\right)+\left\|\nabla \theta^{0}\right\|_{L^{2}\left(\mathbb{T}^{3}\right)}^{2}+\left\|\nabla u^{0}\right\|_{L^{2}\left(\mathbb{T}^{3}\right)}^{2}+\alpha^{2}\left\|\Delta u^{0}\right\|_{L^{2}\left(\mathbb{T}^{3}\right)}^{2},
$$

and $\mu=\min (\nu, \kappa)$. Here, the function $K(t)$ stands for

$$
K(t)=\left\|u^{0}\right\|_{L^{2}}^{2}+\alpha^{2}\left\|\nabla u^{0}\right\|_{L^{2}}^{2}+\left\|\theta^{0}\right\|_{L^{2}}^{2}+\phi_{\alpha}(t)
$$

and $\phi_{\alpha}$ is a positive increasing function of time $t$, defined by

$$
\phi_{\alpha}(t)=\left(\left\|u^{0}\right\|_{L^{2}}^{2}+\alpha^{2}\left\|\nabla u^{0}\right\|_{L^{2}}^{2}+\left\|\theta^{0}\right\|_{L^{2}}^{2}\right)\left(e^{2 t}-1\right)
$$

The proof is based on a Galerkin approximation scheme. While trying to close the energy estimates, the buoyancy force presents some difficulties that we overcome by a Gronwall's type technique. After that, we run a compactness method based on Aubin-Lions lemma [11].

Our second result is a convergence theorem, as $\alpha \rightarrow 0$ :

Theorem 1.2. Let $T>0, u^{0} \in \dot{H}^{2}\left(\mathbb{T}^{3}\right)$ a divergence free vector field, $\theta^{0} \in \dot{H}^{1}\left(\mathbb{T}^{3}\right)$ and $\left(u_{\alpha}, \theta_{\alpha}\right)$, the solution in $[0, T]$ of system (1.10) and $v_{\alpha}=u_{\alpha}-\alpha^{2} \Delta u_{\alpha}$ subject of Theorem 1.1. Then, there exists a time $T^{*}$ such that $0<T^{*} \leq T$ and subsequences $u_{\alpha_{k}}, v_{\alpha_{k}}, \theta_{\alpha_{k}}$, a scalar function $\theta$ and a divergence free vector field $u$ belonging both of them to $L^{\infty}\left(\left[0, T^{*}\right], \dot{H}^{1}\left(\mathbb{T}^{3}\right)\right) \cap L^{2}\left(\left[0, T^{*}\right], \dot{H}^{2}\left(\mathbb{T}^{3}\right)\right)$ such that as $\alpha_{k} \rightarrow 0^{+}$, one has

(1) $u_{\alpha_{k}}$ converges to $u$ and $\theta_{\alpha_{k}}$ converges to $\theta$ weakly in $L^{2}\left(\left[0, T^{*}\right], \dot{H}^{2}\left(\mathbb{T}^{3}\right)\right)$ and strongly in $L^{2}\left(\left[0, T^{*}\right], \dot{H}^{1}\left(\mathbb{T}^{3}\right)\right)$.

(2) $v_{\alpha_{k}}$ converges to $u$ weakly in $L^{2}\left(\left[0, T^{*}\right], \dot{H}^{1}\left(\mathbb{T}^{3}\right)\right)$ and converges strongly in $L^{2}\left(\left[0, T^{*}\right], L^{2}\left(\mathbb{T}^{3}\right)\right)$.

(3) $u_{\alpha_{k}}(t)$ converges to $u(t)$ and $\theta_{\alpha_{k}}(t)$ converges to $\theta(t)$ weakly in $\dot{H}^{1}\left(\mathbb{T}^{3}\right)$ and uniformly over $\left[0, T^{*}\right]$. Furthermore, $(u, \theta)$ is the unique strong solution of the Boussinesq system $(B q)$ on $\left[0, T^{*}\right]$ associated to the initial data $\left(u^{0}, \theta^{0}\right)$ and satisfies, for all $t \in\left[0, T^{*}\right]$, the energy inequality

$$
\begin{aligned}
\|u(t)\|_{L^{2}\left(\mathbb{T}^{3}\right)}^{2}+\|\theta(t)\|_{L^{2}\left(\mathbb{T}^{3}\right)}^{2}+2 \int_{0}^{t} & \nu\|\nabla u(\tau)\|_{L^{2}\left(\mathbb{T}^{3}\right)}^{2}+\kappa\|\nabla \theta(\tau)\|_{L^{2}\left(\mathbb{T}^{3}\right)}^{2} d \tau \\
& \leq\left\|u^{0}\right\|_{L^{2}\left(\mathbb{T}^{3}\right)}^{2}+\left\|\theta^{0}\right\|_{L^{2}\left(\mathbb{T}^{3}\right)}^{2}+2 \phi\left(T^{*}\right) .
\end{aligned}
$$

Here, $(B q)$ and $\phi$ denote respectively (1.10) and $\phi_{\alpha}$, for $\alpha=0$.

Note that the solution of the regularized Lagrangian averaged Boussinesq- $\alpha$ system satisfies an energy inequality that depends on the parameter alpha which provide a singularity, as alpha goes to zero. This is a serious impediment that has to be dealt with when taking the limit as $\alpha_{k} \rightarrow 0$. We shall use a compactness 
method to obtain strong convergence.

The remainder of this paper is divided into two sections; the first is assigned to prove the existence and uniqueness result. The second is concerned by the proofs of convergence results.

\section{WELL-POSEDNESS RESULT}

To study the existence and the regularity of strong solutions. We approximate (1.10) by the following system of ordinary differential equations:

$$
\left\{\begin{array}{l}
\frac{\partial v_{n}}{\partial t}+P_{n} \tilde{B}\left(u_{n}, v_{n}\right)-\nu \triangle v_{n}-\theta_{n} e_{3}=0, \quad(t, x) \in \mathbb{R}_{+} \times \mathbb{T}^{3} \\
\frac{\partial \theta_{n}}{\partial t}-\kappa \triangle \theta_{n}+P_{n} B\left(u_{n}, \theta_{n}\right)=0, \quad(t, x) \in \mathbb{R}_{+} \times \mathbb{T}^{3} \\
v_{n}=\left(1-\alpha^{2} \triangle\right) u_{n}, \quad(t, x) \in \mathbb{R}_{+} \times \mathbb{T}^{3} \\
\operatorname{div} u_{n}=\operatorname{div} v_{n}=0, \quad(t, x) \in \mathbb{R}_{+} \times \mathbb{T}^{3} \\
\left(u_{n}, \theta_{n}\right)_{\mid t=0}=\left(P_{n} u_{0}, P_{n} \theta_{0}\right), \quad x \in \mathbb{T}^{3} .
\end{array}\right.
$$

Both the bilinear operators on the left are continuous on $L^{2} \times L^{2}$. Then, the above system appears as a system of ordinary differential equations on $L^{2}$. Thus, the usual Cauchy-Lipschitz theorem yields the existence of a strictly positive maximal time $T_{n}$ such that a unique solution exists which is continuous in time with value in $L^{2}$. Next, we obtain uniform estimates, with respect to the approximating parameter $n$, on the approximate solutions. To do so, we use conservation laws and product lemmas. Taking the $L^{2}\left(\mathbb{T}^{3}\right)$-inner product of the equation satisfied by $\theta_{n}$ in (2.1) against $-\Delta \theta_{n}$ and the one satisfied by $u_{n}$ against $-\Delta u_{n}$, to obtain

$$
\begin{aligned}
\frac{1}{2} \frac{d}{d t}\left\|\nabla \theta_{n}\right\|_{L^{2}\left(\mathbb{T}^{3}\right)}^{2}+\kappa\left\|\delta \theta_{n}\right\|_{L^{2}\left(\mathbb{T}^{3}\right)}^{2} & =\left\langle B\left(u_{n},, \theta_{n}\right), \Delta \theta_{n}\right\rangle \\
\frac{1}{2} \frac{d}{d t}\left(\left\|\nabla u_{n}\right\|_{L^{2}\left(\mathbb{T}^{3}\right)}^{2}+\alpha^{2}\left\|\Delta u_{n}\right\|_{L^{2}\left(\mathbb{T}^{3}\right)}^{2}\right)+ & \nu\left(\left\|\Delta u_{n}\right\|_{L^{2}\left(\mathbb{T}^{3}\right)}^{2}+\alpha^{2}\left\|\nabla \Delta u_{n}\right\|_{L^{2}\left(\mathbb{T}^{3}\right)}^{2}\right) \\
& =\left\langle\tilde{B}\left(u_{n}, v_{n}\right), \Delta u_{n}\right\rangle+\left\langle\theta_{n} e_{3},-\Delta u_{n}\right\rangle .
\end{aligned}
$$

Now, let us first estimate the right hand sides of (2.2) and (2.3). To do so, we recall the following Sobolev inequalities [11]: for every $\vartheta \in \dot{H}^{1}\left(\mathbb{T}^{3}\right)$, we have

$$
\|\vartheta\|_{L^{3}} \quad \leq\|\vartheta\|_{L^{2}}^{1 / 2}\|\vartheta\|_{\dot{H}^{1}}^{1 / 2}
$$

and

$$
\|\vartheta\|_{L^{6}} \leq c\|\vartheta\|_{\dot{H}^{1}}
$$

Using Hölder's inequality, (2.4) and (2.5), it holds that

$$
\begin{aligned}
\left|\left\langle B\left(u_{n}, \theta_{n}\right), \Delta \theta_{n}\right\rangle\right| & \leq\left\|u_{n}\right\|_{L^{6}\left(\mathbb{T}^{3}\right)}\left\|\nabla \theta_{n}\right\|_{L^{3}\left(\mathbb{T}^{3}\right)}\left\|\Delta \theta_{n}\right\|_{L^{2}\left(\mathbb{T}^{3}\right)} \\
& \leq c\left\|u_{n}\right\|_{\dot{H}^{1}}\left\|\nabla \theta_{n}\right\|_{L^{2}}^{1 / 2}\left\|\nabla \theta_{n}\right\|_{\dot{H}^{1}}^{1 / 2}\left\|\Delta \theta_{n}\right\|_{L^{2}} .
\end{aligned}
$$


Hence, one obtains

$$
\left|\left\langle B\left(u_{n}, \theta_{n}\right), \Delta \theta_{n}\right\rangle\right| \leq c\left\|u_{n}\right\|_{\dot{H}^{1}}\left\|\nabla \theta_{n}\right\|_{L^{2}}^{1 / 2}\left\|\Delta \theta_{n}\right\|_{L^{2}}^{3 / 2} .
$$

For every $\vartheta \in \dot{H}^{2}\left(\mathbb{T}^{3}\right)$, the Agmon's inequality [11] reads

$$
\|\vartheta\|_{L^{\infty}} \leq\|\vartheta\|_{\dot{H}^{1}}^{1 / 2}\|\vartheta\|_{\dot{H}^{2}}^{1 / 2}
$$

The fact that $v_{n}=u_{n}-\alpha^{2} \Delta u_{n}$ yields

$$
\begin{aligned}
\left|\left\langle\tilde{B}\left(u_{n}, v_{n}\right), \Delta u_{n}\right\rangle\right| & =\left|\left\langle B\left(u_{n}, v_{n}\right), \Delta u_{n}\right\rangle-\left\langle B\left(\Delta u_{n}, v_{n}\right), u_{n}\right\rangle\right| \\
\leq & \left|\left\langle B\left(u_{n}, u_{n}\right), \Delta u_{n}\right\rangle\right|+\alpha^{2}\left|\left\langle B\left(u_{n}, \Delta u_{n}\right), \Delta u_{n}\right\rangle\right| \\
+ & \left|\left\langle B\left(\Delta u_{n}, u_{n}\right), u_{n}\right\rangle\right|+\alpha^{2}\left|\left\langle B\left(\Delta u_{n}, \Delta u_{n}\right), u_{n}\right\rangle\right| .
\end{aligned}
$$

The first nonlinear term is to be dealt with as follows:

$$
\begin{aligned}
\left|\left\langle B\left(u_{n}, u_{n}\right), \Delta u_{n}\right\rangle\right| & \leq\left\|u_{n}\right\|_{L^{\infty}\left(\mathbb{T}^{3}\right)}\left\|\nabla u_{n}\right\|_{L^{2}\left(\mathbb{T}^{3}\right)}\left\|\Delta u_{n}\right\|_{L^{2}\left(\mathbb{T}^{3}\right)} \\
& \leq c\left\|u_{n}\right\|_{\dot{H}^{1}}^{1 / 2}\left\|u_{n}\right\|_{\dot{H}^{2}}^{1 / 2}\left\|\nabla u_{n}\right\|_{L^{2}\left(\mathbb{T}^{3}\right)}\left\|\Delta u_{n}\right\|_{L^{2}\left(\mathbb{T}^{3}\right)} \\
& \leq c\left\|u_{n}\right\|_{\dot{H}^{1}}^{1 / 2}\left\|\nabla u_{n}\right\|_{L^{2}\left(\mathbb{T}^{3}\right)}\left\|\Delta u_{n}\right\|_{L^{2}\left(\mathbb{T}^{3}\right)}^{3 / 2} \\
& \leq c\left\|u_{n}\right\|_{\dot{H}^{1}\left(\mathbb{T}^{3}\right)}\left\|\Delta u_{n}\right\|_{L^{2}\left(\mathbb{T}^{3}\right)}^{1 / 2}\left\|\nabla \Delta u_{n}\right\|_{L^{2}\left(\mathbb{T}^{3}\right)}^{3 / 2},
\end{aligned}
$$

where we used Hölder's inequality, inequality (2.7) and the facts that $\left\|\nabla u_{n}\right\|_{L^{2}} \leq\left\|\Delta u_{n}\right\|_{L^{2}}$ and $\left\|\Delta u_{n}\right\|_{L^{2}} \leq$ $\left\|\nabla \Delta u_{n}\right\|_{L^{2}}$. Similarly, we have

$$
\left|\left\langle B\left(\Delta u_{n}, u_{n}\right), u_{n}\right\rangle\right| \leq c\left\|u_{n}\right\|_{\dot{H}^{1}\left(\mathbb{T}^{3}\right)}\left\|\Delta u_{n}\right\|_{L^{2}\left(\mathbb{T}^{3}\right)}^{1 / 2}\left\|\nabla \Delta u_{n}\right\|_{L^{2}\left(\mathbb{T}^{3}\right)}^{3 / 2} .
$$

The second nonlinear term is to be dealt with, in the following manner:

$$
\begin{aligned}
\left|\left\langle B\left(u_{n}, \Delta u_{n}\right), \Delta u_{n}\right\rangle\right| \leq\left\|u_{n}\right\|_{L^{6}\left(\mathbb{T}^{3}\right)}\left\|\nabla \Delta u_{n}\right\|_{L^{2}\left(\mathbb{T}^{3}\right)}\left\|\Delta u_{n}\right\|_{L^{3}\left(\mathbb{T}^{3}\right)} \\
\leq c\left\|u_{n}\right\|_{\dot{H}^{1}}\left\|\nabla \Delta u_{n}\right\|_{L^{2}\left(\mathbb{T}^{3}\right)}\left\|\Delta u_{n}\right\|_{L^{2}\left(\mathbb{T}^{3}\right)}^{1 / 2}\left\|\nabla \Delta u_{n}\right\|_{L^{2}\left(\mathbb{T}^{3}\right)}^{1 / 2} \\
=c\left\|u_{n}\right\|_{\dot{H}^{1}\left(\mathbb{T}^{3}\right)}\left\|\Delta u_{n}\right\|_{L^{2}\left(\mathbb{T}^{3}\right)}^{1 / 2}\left\|\nabla \Delta u_{n}\right\|_{L^{2}\left(\mathbb{T}^{3}\right)}^{3 / 2} .
\end{aligned}
$$

Similarly, we have

$$
\begin{aligned}
\left|\left\langle B\left(\Delta u_{n}, \Delta u_{n}\right), u_{n}\right\rangle\right| & \leq\left\|\Delta u_{n}\right\|_{L^{3}\left(\mathbb{T}^{3}\right)}\left\|\nabla \Delta u_{n}\right\|_{L^{2}\left(\mathbb{T}^{3}\right)}\left\|u_{n}\right\|_{L^{6}\left(\mathbb{T}^{3}\right)} \\
& \leq c\left\|u_{n}\right\|_{\dot{H}^{1}\left(\mathbb{T}^{3}\right)}\left\|\Delta u_{n}\right\|_{L^{2}\left(\mathbb{T}^{3}\right)}^{1 / 2}\left\|\nabla \Delta u_{n}\right\|_{L^{2}\left(\mathbb{T}^{3}\right)}^{3 / 2} .
\end{aligned}
$$

It turns out that

$$
\left|\left\langle\tilde{B}\left(u_{n}, v_{n}\right), \Delta u_{n}\right\rangle\right| \leq c\left(1+\alpha^{2}\right)\left\|u_{n}\right\|_{\dot{H}^{1}\left(\mathbb{T}^{3}\right)}\left\|\Delta u_{n}\right\|_{L^{2}\left(\mathbb{T}^{3}\right)}^{1 / 2}\left\|\nabla \Delta u_{n}\right\|_{L^{2}\left(\mathbb{T}^{3}\right)}^{3 / 2}
$$

By Cauchy-Schwarz and Young's inequalities, we get

$$
\left|\left\langle\theta_{n} e_{3},-\Delta u_{n}\right\rangle\right| \leq\left\|\nabla \theta_{n}\right\|_{L^{2}}^{2}+\left\|\nabla u_{n}\right\|_{L^{2}}^{2} .
$$


Summing up (2.2) and (2.3) and using (2.6), (2.8) and (2.9), it follows that

$$
\begin{gathered}
\frac{1}{2} \frac{d}{d t}\left(\left\|\nabla \theta_{n}\right\|_{L^{2}\left(\mathbb{T}^{3}\right)}^{2}+\left\|\nabla u_{n}\right\|_{L^{2}\left(\mathbb{T}^{3}\right)}^{2}+\alpha^{2}\left\|\Delta u_{n}\right\|_{L^{2}\left(\mathbb{T}^{3}\right)}^{2}\right) \\
+\kappa\left\|\Delta \theta_{n}\right\|_{L^{2}\left(\mathbb{T}^{3}\right)}^{2}+\nu\left(\left\|\Delta u_{n}\right\|_{L^{2}\left(\mathbb{T}^{3}\right)}^{2}+\alpha^{2}\left\|\nabla \Delta u_{n}\right\|_{L^{2}\left(\mathbb{T}^{3}\right)}^{2}\right) \\
\leq c\left\|u_{n}\right\|_{\dot{H}^{1}}\left\|\nabla \theta_{n}\right\|_{L^{2}}^{1 / 2}\left\|\Delta \theta_{n}\right\|_{L^{2}}^{3 / 2}+\left\|\nabla \theta_{n}\right\|_{L^{2}}^{2}+\left\|\nabla u_{n}\right\|_{L^{2}}^{2} \\
\quad c\left(1+\alpha^{2}\right)\left\|u_{n}\right\|_{\dot{H}^{1}\left(\mathbb{T}^{3}\right)}\left\|\Delta u_{n}\right\|_{L^{2}\left(\mathbb{T}^{3}\right)}^{1 / 2}\left\|\nabla \Delta u_{n}\right\|_{L^{2}\left(\mathbb{T}^{3}\right)}^{3 / 2} .
\end{gathered}
$$

Using Young's inequality, we absorb the remaining diffusion term in the right hand side and we obtain

$$
\begin{array}{r}
\frac{d}{d t}\left(\left\|\nabla \theta_{n}\right\|_{L^{2}}^{2}+\left\|\nabla u_{n}\right\|_{L^{2}}^{2}+\alpha^{2}\left\|\Delta u_{n}\right\|_{L^{2}}^{2}\right) \\
+\kappa\left\|\Delta \theta_{n}\right\|_{L^{2}}^{2}+\nu\left(\left\|\Delta u_{n}\right\|_{L^{2}}^{2}+\alpha^{2}\left\|\nabla \Delta u_{n}\right\|_{L^{2}}^{2}\right) \\
\leq c\left\|u_{n}\right\|_{\dot{H}^{1}}^{4}\left\|\nabla \theta_{n}\right\|_{L^{2}}^{2}+\left\|\nabla \theta_{n}\right\|_{L^{2}}^{2}+\left\|\nabla u_{n}\right\|_{L^{2}}^{2},
\end{array}
$$

where $c$ is a generic constant that may change from line to line. Integrating over time, we obtain, for all $t \in\left[0, T_{n}^{*}\right)$,

$$
\begin{array}{r}
\left\|\nabla \theta_{n}(t)\right\|_{L^{2}\left(\mathbb{T}^{3}\right)}^{2}+\left\|\nabla u_{n}(t)\right\|_{L^{2}\left(\mathbb{T}^{3}\right)}^{2}+\alpha^{2}\left\|\Delta u_{n}(t)\right\|_{L^{2}\left(\mathbb{T}^{3}\right)}^{2}+\kappa \int_{0}^{t}\left\|\Delta \theta_{n}(\tau)\right\|_{L^{2}}^{2} d \tau \\
+\nu \int_{0}^{t}\left(\left\|\Delta u_{n}(\tau)\right\|_{L^{2}\left(\mathbb{T}^{3}\right)}^{2}+\alpha^{2}\left\|\nabla \Delta u_{n}(\tau)\right\|_{L^{2}\left(\mathbb{T}^{3}\right)}^{2}\right) d \tau \\
\leq c \int_{0}^{t}\left\|u_{n}(\tau)\right\|_{\dot{H}^{1}}^{4}\left(\left\|\Delta u_{n}(\tau)\right\|_{L^{2}}^{2}+\left\|\nabla \theta_{n}(\tau)\right\|_{L^{2}}^{2}\right) d \tau \\
+\int_{0}^{t}\left(\left\|\nabla \theta_{n}(\tau)\right\|_{L^{2}}^{2}+\left\|\nabla u_{n}(\tau)\right\|_{L^{2}}^{2}\right) d \tau+\left\|\nabla \theta^{0}\right\|_{L^{2}}^{2}+\left\|\nabla u^{0}\right\|_{L^{2}}^{2}+\alpha^{2}\left\|\Delta u^{0}\right\|_{L^{2}}^{2}
\end{array}
$$

At this point, we give the theorem below that states the existence weak solution. The proof follows exactly the lines of the proof given in [12]:

Theorem 2.1. Let $\theta_{0} \in L^{2}\left(\mathbb{T}^{3}\right)$, and $u_{0} \in H^{1}\left(\mathbb{T}^{3}\right)$ be a divergence-free vector field. Then, for any $T>0$ there exists a unique weak solution $\left(u_{\alpha}, \theta_{\alpha}\right)$ to (1.1) in the interval $[0, T]$, where

$$
u_{\alpha} \in C\left([0, T], H^{1}\left(\mathbb{T}^{3}\right)\right) \cap L^{2}\left([0, T], H^{2}\left(\mathbb{T}^{3}\right)\right),
$$

and

$$
\theta_{\alpha} \in C\left([0, T], L^{2}\left(\mathbb{T}^{3}\right)\right) \cap L^{2}\left([0, T], H^{1}\left(\mathbb{T}^{3}\right)\right) .
$$

Moreover, we have for all $t \in[0, T]$ :

$$
\begin{array}{r}
\left(\|u(t)\|_{L^{2}\left(\mathbb{T}^{3}\right)}^{2}+\alpha^{2}\|\nabla u(t)\|_{L^{2}\left(\mathbb{T}^{3}\right)}^{2}+\|\theta(t)\|_{L^{2}\left(\mathbb{T}^{3}\right)}^{2}\right) \\
+2 \mu \int_{0}^{t}\left(\|\nabla u(\tau)\|_{L^{2}\left(\mathbb{T}^{3}\right)}^{2}+\alpha^{2}\|\Delta u(\tau)\|_{L^{2}\left(\mathbb{T}^{3}\right)}^{2}+\|\nabla \theta(\tau)\|_{L^{2}\left(\mathbb{T}^{3}\right)}^{2}\right) d \tau \\
\leq \underbrace{\left\|u_{0}\right\|_{L^{2}}^{2}+\alpha^{2}\left\|\nabla u_{0}\right\|_{L^{2}}^{2}+\left\|\theta_{0}\right\|_{L^{2}}^{2}+\phi_{\alpha}(t)}_{K(t)}
\end{array}
$$

where by $\phi_{\alpha}(t)$ we refer to the function $\left(\left\|u_{0}\right\|_{L^{2}}^{2}+\alpha^{2}\left\|\nabla u_{0}\right\|_{L^{2}}^{2}+\left\|\theta_{0}\right\|_{L^{2}}^{2}\right)\left(e^{2 t}-1\right)$. Furthermore, this solution is continuously dependent on the initial data $\left(u_{0}, \theta_{0}\right)$. Moreover, this solution is continuously dependent on the initial data $\left(u_{0}, \theta_{0}\right)$. In particular, it is unique. 
Using the energy estimate for weak solution $\left(u_{\alpha}, \theta_{\alpha}\right)$ in theorem above, and the expression of the function $\rho_{\alpha}$ given by equation (1.12), we infer that

$$
\int_{0}^{t}\left(\left\|\nabla \theta_{n}(\tau)\right\|_{L^{2}}^{2}+\left\|\nabla u_{n}(\tau)\right\|_{L^{2}}^{2}\right) d \tau \leq \frac{K(T)}{2 \min (\nu, \kappa)}
$$

and

$$
\begin{aligned}
\int_{0}^{t}\left\|u_{n}\right\|_{\dot{H}^{1}}^{4}\left(\left\|\Delta u_{n}\right\|_{L^{2}}^{2}+\left\|\nabla \theta_{n}\right\|_{L^{2}}^{2}\right) d \tau & \leq\left\|u_{n}\right\|_{L_{T}^{\infty}\left(\dot{H}^{1}\right)}^{4}\left(\left\|\theta_{n}\right\|_{L_{T}^{2}\left(\dot{H}^{1}\right)}^{2}+\left\|u_{n}\right\|_{L_{T}^{2}\left(\dot{H}^{2}\right)}^{2}\right) \\
& \leq \frac{K^{2}(T)}{\alpha^{4}}\left(\frac{K(T)}{2 \kappa}+\frac{K(T)}{2 \nu \alpha^{2}}\right) \leq \frac{K^{3}(T)\left(1+\alpha^{2}\right)}{2 \min (\nu, \kappa) \alpha^{6}}
\end{aligned}
$$

where $K(t)=\left\|u_{n}^{0}\right\|_{L^{2}}^{2}+\alpha^{2}\left\|\nabla u_{n}^{0}\right\|_{L^{2}}^{2}+\left\|\theta_{n}^{0}\right\|_{L^{2}}^{2}+\phi_{\alpha}(T)$.

In conclusion, we obtain the energy estimate

$$
\begin{aligned}
\left\|\nabla \theta_{n}(t)\right\|_{L^{2}}^{2} & +\left\|\nabla u_{n}(t)\right\|_{L^{2}}^{2}+\alpha^{2}\left\|\Delta u_{n}(t)\right\|_{L^{2}}^{2}+\kappa \int_{0}^{t}\left\|\Delta \theta_{n}(\tau)\right\|_{L^{2}}^{2} d \tau \\
& +\nu \int_{0}^{t}\left(\left\|\Delta u_{n}(\tau)\right\|_{L^{2}}^{2}+\alpha^{2}\left\|\Delta \nabla u_{n}(\tau)\right\|_{L^{2}}^{2}\right) d \tau \leq \mathcal{K}_{\alpha}(T)
\end{aligned}
$$

where

$$
\mathcal{K}_{\alpha}(T)=\frac{K(T)}{2 \mu}+\frac{K^{3}(T)}{2 \mu \alpha^{6}}\left(1+\alpha^{2}\right)+\left\|\nabla \theta^{0}\right\|_{L^{2}\left(\mathbb{T}^{3}\right)}^{2}+\left\|\nabla u^{0}\right\|_{L^{2}\left(\mathbb{T}^{3}\right)}^{2}+\alpha^{2}\left\|\Delta u^{0}\right\|_{L^{2}\left(\mathbb{T}^{3}\right)}^{2}
$$

where by $\mu=\min (\nu, \kappa)$.

Notice that the upper bound $\mathcal{K}(t)$ in $(2.11)$ is continuous and does not include any singularity with respect to time $t$. Hence, $\mathcal{K}(t)$ rules out the finite time blow-up of the solution near $T$ and the solution can be extended to a global in time solution. The estimation (2.11) provides uniform bounds, with respect to $n$, of the solution $u_{n}$ in $L_{T}^{\infty}\left(\dot{H}^{2}\left(\mathbb{T}^{3}\right)\right), L_{T}^{2}\left(\dot{H}^{3}\left(\mathbb{T}^{3}\right)\right)$ as well as in $L_{T}^{\infty}\left(\dot{H}^{1}\left(\mathbb{T}^{3}\right)\right)$ and $L_{T}^{2}\left(\dot{H}^{2}\left(\mathbb{T}^{3}\right)\right)$ for $\theta_{n}$. This allows to use the Aubin-Lions lemma so that we can take the limit as $n$ tends to infinity and then obtain existence. As strong solutions are also weak, uniqueness of strong solution simply follows from uniqueness of weak solution.

\section{Convergence Result}

Already, we have proved that an initial data $\left(u^{0}, \theta^{0}\right)$ gives rise to a global solution $\left(u_{\alpha}, \theta_{\alpha}\right)$. This section is aimed to deal with the convergence result, as the parameter $\alpha$ vanishes. It must be said that the upper bound in the energy estimate (2.11) depends singularly on $\alpha$ and it will fail to control the solution's norms, as $\alpha$ goes to zero. From (2.10), it is worth mentioning that the dependence of the upper bound in the weak solution's energy estimate is polynomial, so that such impediment is absent in the weak solution case. To overcome this difficulty, we need to get uniform bounds, with respect to $\alpha$ on

$$
\left\|\nabla \theta_{\alpha}(t)\right\|_{L^{2}\left(\mathbb{T}^{3}\right)}^{2}+\left\|\nabla u_{\alpha}(t)\right\|_{L^{2}\left(\mathbb{T}^{3}\right)}^{2}+\alpha^{2}\left\|\Delta u_{\alpha}(t)\right\|_{L^{2}\left(\mathbb{T}^{3}\right)}^{2}
$$


and

$$
\kappa \int_{0}^{t}\left\|\Delta \theta_{\alpha}(\tau)\right\|_{L^{2}}^{2} d \tau+\nu \int_{0}^{t}\left(\left\|\Delta u_{\alpha}(\tau)\right\|_{L^{2}\left(\mathbb{T}^{3}\right)}^{2}+\alpha^{2}\left\|\nabla \Delta u_{\alpha}(\tau)\right\|_{L^{2}\left(\mathbb{T}^{3}\right)}^{2}\right) d \tau .
$$

To do so, firstly we mention that

$$
\begin{array}{r}
\left|\left\langle\tilde{B}\left(u_{\alpha}, v_{\alpha}\right), \Delta u_{\alpha}\right\rangle\right| \leq c\left\|u_{\alpha}\right\|_{\dot{H}^{1}}^{1 / 2}\left\|u_{\alpha}\right\|_{\dot{H}^{2}}^{1 / 2}\left\|\nabla v_{\alpha}\right\|_{L^{2}\left(\mathbb{T}^{3}\right)}\left\|\Delta u_{\alpha}\right\|_{L^{2}\left(\mathbb{T}^{3}\right)} \\
\leq c\left\|u_{\alpha}\right\|_{\dot{H}^{1}}^{1 / 2}\left\|u_{\alpha}\right\|_{\dot{H}^{2}}^{1 / 2}\left(\left\|u_{\alpha}\right\|_{\dot{H}^{1}\left(\mathbb{T}^{3}\right)}+\alpha^{2}\left\|\nabla \Delta u_{\alpha}\right\|_{L^{2}\left(\mathbb{T}^{3}\right)}\right)\left\|\Delta u_{\alpha}\right\|_{L^{2}\left(\mathbb{T}^{3}\right)} \\
\leq c\left(\left\|u_{\alpha}\right\|_{\dot{H}^{1}}^{6}+\alpha^{6}\left\|\Delta u_{\alpha}\right\|_{L^{2}}^{6}\right)+\nu / 2\left\|\Delta u_{\alpha}\right\|_{L^{2}\left(\mathbb{T}^{3}\right)}^{2}+\alpha^{2} \nu / 2\left\|\nabla \Delta u_{\alpha}\right\|_{L^{2}\left(\mathbb{T}^{3}\right)}^{2},
\end{array}
$$

where we used Agmon's inequality and Young's inequality twice.

Secondly, using Hölder inequality and Young's inequality twice, it turns out that

$$
\begin{aligned}
\left|\left\langle\left(u_{\alpha} \cdot \nabla\right) \theta_{\alpha}, \Delta \theta_{\alpha}\right\rangle\right| & \leq c\left\|u_{\alpha}\right\|_{\dot{H}^{1}}^{4}\left\|\nabla \theta_{\alpha}\right\|_{L^{2}}^{2}+\kappa / 2\left\|\Delta \theta_{\alpha}\right\|_{L^{2}}^{2} \\
& \leq c\left(\left\|u_{\alpha}\right\|_{\dot{H}^{1}}^{6}+\left\|\nabla \theta_{\alpha}\right\|_{L^{2}}^{6}\right)+\kappa / 2\left\|\Delta \theta_{\alpha}\right\|_{L^{2}}^{2} .
\end{aligned}
$$

Thirdly, the bouancy force can be dealt with via Cauchy-Schwarz inequality:

$$
\left|\left\langle\theta_{\alpha} e_{3},-\Delta u_{\alpha}\right\rangle\right| \leq\left\|\nabla \theta_{\alpha}\right\|_{L^{2}}^{2}+\left\|\nabla u_{\alpha}\right\|_{L^{2}}^{2}+\alpha^{2}\left\|\Delta u_{\alpha}\right\|_{L^{2}}^{2} .
$$

Finally, summing up, one obtains

$$
\begin{aligned}
& \frac{1}{2} \frac{d}{d t}\left(\left\|\nabla \theta_{\alpha}\right\|_{L^{2}\left(\mathbb{T}^{3}\right)}^{2}+\left\|\nabla u_{\alpha}\right\|_{L^{2}\left(\mathbb{T}^{3}\right)}^{2}+\alpha^{2}\left\|\Delta u_{\alpha}\right\|_{L^{2}\left(\mathbb{T}^{3}\right)}^{2}\right) \\
& +\kappa / 2\left\|\Delta \theta_{\alpha}\right\|_{L^{2}\left(\mathbb{T}^{3}\right)}^{2}+\nu / 2\left(\left\|\Delta u_{\alpha}\right\|_{L^{2}\left(\mathbb{T}^{3}\right)}^{2}+\alpha^{2}\left\|\nabla \Delta u_{\alpha}\right\|_{L^{2}\left(\mathbb{T}^{3}\right)}^{2}\right) \\
& \leq c\left(\left\|\nabla \theta_{\alpha}\right\|_{L^{2}}^{6}+\left\|\nabla u_{\alpha}\right\|_{L^{2}}^{6}+\alpha^{6}\left\|\Delta u_{\alpha}\right\|_{L^{2}}^{6}\right. \\
& \left.+\left\|\nabla \theta_{\alpha}\right\|_{L^{2}}^{2}+\left\|\nabla u_{\alpha}\right\|_{L^{2}}^{2}+\alpha^{2}\left\|\Delta u_{\alpha}\right\|_{L^{2}}^{2}\right),
\end{aligned}
$$

where $c$ is a constant that does not depend on the parameter $\alpha$. Let $g(t)=\left\|\nabla \theta_{\alpha}(t)\right\|_{L^{2}\left(\mathbb{T}^{3}\right)}^{2}+\left\|\nabla u_{\alpha}(t)\right\|_{L^{2}\left(\mathbb{T}^{3}\right)}^{2}+$ $\alpha^{2}\left\|\Delta u_{\alpha}(t)\right\|_{L^{2}\left(\mathbb{T}^{3}\right)}^{2}$. It is clear that $g^{3}+g \leq c(g+1)^{3}$. Let $h(t)=g(t)+1$. The function $g$ is a non-negative function, so $h(0) \neq 0$. The estimation (3.1) can be written as $\frac{d h}{d t} \leq c h^{3}$. We integrate this ordinary differential inequality, to obtain for $0 \leq t \leq \frac{1}{4 C h^{2}(0)}$,

$$
h(t) \leq \operatorname{ch}(0)
$$

Finally, it turns out that for all time $t$, such that

$$
0 \leq t \leq T^{*}=\min \left(T, \frac{1}{4 C(1+g(0))^{2}}\right)
$$

we have

$$
\begin{aligned}
& \left\|\nabla \theta_{\alpha}\right\|_{L^{2}\left(\mathbb{T}^{3}\right)}^{2}+\left\|\nabla u_{\alpha}\right\|_{L^{2}\left(\mathbb{T}^{3}\right)}^{2}+\alpha^{2}\left\|\Delta u_{\alpha}\right\|_{L^{2}\left(\mathbb{T}^{3}\right)}^{2} \\
& \leq c\left(1+\left\|\nabla \theta^{0}\right\|_{L^{2}\left(\mathbb{T}^{3}\right)}^{2}+\left\|\nabla u^{0}\right\|_{L^{2}\left(\mathbb{T}^{3}\right)}^{2}+\alpha^{2}\left\|\Delta u^{0}\right\|_{L^{2}\left(\mathbb{T}^{3}\right)}^{2}\right) .
\end{aligned}
$$


Integrating (3.1) over $\left(0, T^{*}\right)$ and using (3.2), we obtain

$$
\begin{array}{r}
\int_{0}^{T^{*}}\left(\kappa\left\|\Delta \theta_{\alpha}\right\|_{\left.L^{2}\right)}^{2}+\nu\left(\left\|\Delta u_{\alpha}\right\|_{L^{2}}^{2}+\alpha^{2}\left\|\nabla \Delta u_{\alpha}\right\|_{L^{2}}^{2}\right) d t\right. \\
\leq c\left(1+\left\|\nabla \theta^{0}\right\|_{L^{2}\left(\mathbb{T}^{3}\right)}^{2}+\left\|\nabla u^{0}\right\|_{L^{2}\left(\mathbb{T}^{3}\right)}^{2}+\alpha^{2}\left\|\Delta u^{0}\right\|_{L^{2}\left(\mathbb{T}^{3}\right)}^{2}\right) .
\end{array}
$$

These are non singular bounds with respect to the parameter $\alpha$. Since $\alpha$ is intended to vanish, then there exists some fixed value $\alpha_{0}$, such that $0<\alpha \leq \alpha_{0}$. We take $\alpha=\alpha_{0}$ in (3.2) and (3.3), to obtain a uniform bound with respect to $\alpha$. Namely, the functions $\theta$ and $u$ are uniformly bounded in $L^{2}\left(\left[0, T^{*}\right], \dot{H}^{2}\left(\mathbb{T}^{3}\right)\right)$, as for $v$, it is uniformly bounded in $L^{2}\left(\left[0, T^{*}\right], \dot{H}^{1}\left(\mathbb{T}^{3}\right)\right)$. Hence, Banach-Alaoglu theorem [11] allows to extract subsequences $\left(u_{\alpha_{k}}\right)_{k},\left(v_{\alpha_{k}}\right)_{k}$ and $\left(\theta_{\alpha_{k}}\right)_{k}$ (that we relabel $\left(u_{k}\right),\left(v_{k}\right)$ and $\left.\left(\theta_{k}\right)\right)$ respectively of $u_{\alpha}, v_{\alpha}$ and $\theta_{\alpha}$ such that $\left(\theta_{k}, u_{k}\right) \rightarrow(\theta, u)$ in $L^{2}\left(\left[0, T^{*}\right], \dot{H}^{2}\left(\mathbb{T}^{3}\right)\right)$ and $v_{k} \rightarrow u$ in $L^{2}\left(\left[0, T^{*}\right], \dot{H}^{1}\left(\mathbb{T}^{3}\right)\right)$, as $k \rightarrow+\infty$. At this step, we proved the two first results of statements 1 and 2 of Theorem 1.2.

To investigate the two second results of these statements, we establish uniform estimates, independent of $\alpha$, for $\frac{d}{d t} \theta_{\alpha_{k}}$ and $\frac{d}{d t} u_{\alpha_{k}}$. For a fixed positive time, since $\theta_{\alpha_{k}}$ is uniformly bounded with respect to $\alpha$, in $L^{2}\left(\left[0, T^{*}\right], \dot{H}^{2}\left(\mathbb{T}^{3}\right)\right)$, the diffusion $\Delta \theta_{\alpha_{k}}$ belongs to $L^{2}\left(\left[0, T^{*}\right], L^{2}\left(\mathbb{T}^{3}\right)\right)$. Using Sobolev norm definition and product laws we infer that

$$
\int_{0}^{T^{*}}\left\|\operatorname{div} \theta_{k} u_{k}\right\|_{L^{2}}^{2} d \tau \leq \int_{0}^{T^{*}}\left\|\theta_{k}\right\|_{\dot{H}^{1}}^{2}\left\|u_{k}\right\|_{\dot{H}^{2}}^{2} d \tau \leq\left\|\theta_{k}\right\|_{L_{T^{*}}^{\infty}\left(\dot{H}^{1}\right)}^{2}\left\|u_{k}\right\|_{L_{T^{*}}^{2}\left(\dot{H}^{2}\right)^{2}}^{2}
$$

Since $u_{k}$ and $\theta_{k}$ are, respectively, subsequences of $u_{\alpha}$ and $\theta_{\alpha}$, then the energy estimates (3.2) and (3.3) apply also for $u_{k}$ and $\theta_{k}$ and one can control the advection in $L^{2}\left(\left[0, T^{*}\right], L^{2}\left(\mathbb{T}^{3}\right)\right)$. The above temperature diffusion and convection estimates lead to $\left\|\frac{d}{d t} \theta_{k}\right\|_{L_{T^{*}}^{2}\left(L^{2}\right)} \leq K_{1}$, where $K_{1}$ is a real positive constant. To handle the time derivative of the velocity field $u_{k}$, we apply the Helmholtz operator $\left(I-\alpha^{2} \Delta\right)^{-1}$ on the equation satisfied by $u=u_{k}$ in the system (1.10). So,

$$
\begin{aligned}
\frac{d}{d t} u_{k} & =\nu \Delta u_{k}-\left(I-\alpha^{2} \Delta\right)^{-1} \tilde{B}\left(u_{k}, v_{k}\right) \\
& -\left(I-\alpha^{2} \Delta\right)^{-1} \nabla p_{k}+\left(I-\alpha^{2} \Delta\right)^{-1} \theta_{k} e_{3} .
\end{aligned}
$$

For a fixed positif time, since $u_{k}$ is uniformly bounded with respect to $\alpha$ in the space $L^{2}\left(\left[0, T^{*}\right], \dot{H}^{2}\left(\mathbb{T}^{3}\right)\right)$, then $\Delta u_{k}$ belongs to $L^{2}\left(\left[0, T^{*}\right], L^{2}\left(\mathbb{T}^{3}\right)\right)$. For the remaining terms, we recall that operator $\left(I-\alpha^{2} \Delta\right)^{-1}$ is bounded from $H^{-2}\left(\mathbb{T}^{3}\right)$ into $L^{2}\left(\mathbb{T}^{3}\right)$. Moreover, a direct frequencies computation implies that its norm is uniformly bounded and satisfies

$$
\left\|\left(I-\alpha^{2} \Delta\right)^{-1} \mid\right\| \leq 1
$$


Since $\theta_{\alpha} \in L_{T^{*}}^{2}\left(\dot{H}^{2}\right)$, one infers that $\left\|\left(I-\alpha^{2} \Delta\right)^{-1} \theta e_{3}\right\|_{L_{T^{*}}^{2}\left(\dot{H}^{4}\right)} \leq K_{2}^{\prime}$, where $K_{2}^{\prime}$ is a real positif constant. To estimate the nonlinear term, one has

$$
\begin{array}{r}
\int_{0}^{T^{*}}\left\|\left(I-\alpha^{2} \Delta\right)^{-1} \tilde{B}\left(u_{k}, v_{k}\right)\right\|_{L^{2}}^{2} \leq \int_{0}^{T^{*}}\left\|\tilde{B}\left(u_{k}, v_{k}\right)\right\|_{H^{-2}}^{2} \leq c\left\|u_{k}\right\|_{L_{T^{*}}^{\infty}\left(\dot{H}^{1}\right)}^{2}\left\|v_{k}\right\|_{L_{T^{*}}^{2}\left(\dot{H}^{1}\right)}^{2} \\
\leq c\left\|u_{k}\right\|_{L_{T^{*}}^{\infty}\left(\dot{H}^{1}\right)}^{2}\left(\int_{0}^{T^{*}}\left\|\Delta u_{k}(\tau)\right\|_{L^{2}}^{2}+\alpha^{2}\left\|\nabla \Delta u_{k}(\tau)\right\|_{L^{2}}^{2} d \tau\right) .
\end{array}
$$

Inequalities (3.2) and (3.3) provide uniform bounds to the non-linearity above. Using precedent uniform bounds, thanks to the divergence free-condition, one infers that $\left\|\left(I-\alpha^{2} \Delta\right)^{-1} \nabla p\right\|_{L_{T^{*}}^{2}\left(\dot{H}^{4}\right)} \leq K_{2}^{\prime \prime}$. So, equation (3.4) implies that

$$
\left\|\frac{d}{d t} u_{k}\right\|_{L_{T^{*}}^{2}\left(L^{2}\right)} \leq K_{2}
$$

Using Aubin-Lions lemma, we extract two subsequences relabeled $u_{k}$ and $\theta_{k}$, that converge strongly in $L^{2}\left(\left[0, T^{*}\right], \dot{H}^{1}\right)$ and in $L^{2}\left(\left[0, T^{*}\right], L^{2}\right)$, respectively. We have

$$
\left\|v_{k}-u_{k}\right\|_{L^{2}\left(\left[0, T^{*}\right], L^{2}\right)}^{2}=\alpha^{4} \int_{0}^{T}\left(\sum_{k \in \mathbb{Z}^{3}}\left|\widehat{\Delta u_{k}}\right|^{2}\right)=\alpha^{4}\left\|u_{k}\right\|_{L^{2}\left(\left[0, T^{*}\right], \dot{H}^{2}\right)}^{2} .
$$

As $u_{k}$ belongs to $L^{2}\left(\left[0, T^{*}\right], \dot{H}^{2}\right), v_{k}$ converges strongly to $u$ in $L^{2}\left(\left[0, T^{*}\right], L^{2}\right)$, we have already proved the statements 1 and 2 of Theorem 1.2.

Now, we turn to the third statement of Theorem 1.2. For the first result, since $\left(u_{k}, \theta_{k}\right)$ converges strongly to $(u, \theta)$ in $\left(L^{2}\left(\left[0, T^{*}\right], \dot{H}^{1}\right)\right)^{2}$, then by Cauchy-Schwarz inequality it converges weakly for almost every $t \in\left[0, T^{*}\right]$. In particular, this holds for the supremum. That is $\left(u_{k}(t), \theta_{k}(t)\right)$ converges to $(u(t), \theta(t))$ weakly in $\dot{H}^{1}\left(\mathbb{T}^{3}\right)$ and uniformly over $\left[0, T^{*}\right]$. To prove the second result, using the precedent bounds of time derivatives, Banach-Alaoglu theorem in Hilbert spaces implies that

$$
\left(\frac{d}{d t} \theta_{k}, \frac{d}{d t} u_{k}\right) \rightarrow\left(\frac{d}{d t} \theta, \frac{d}{d t} u\right) \text { weakly in } L^{2}\left(\left[0, T^{*}\right], L^{2}\left(\mathbb{T}^{3}\right)\right), \text { as } k \rightarrow+\infty,
$$

and

$$
\frac{d}{d t} v_{k} \rightarrow \frac{d}{d t} u \text { weakly in } L^{2}\left(\left[0, T^{*}\right], \dot{H}^{-2}\left(\mathbb{T}^{3}\right)\right) \text {, as } k \rightarrow+\infty \text {. }
$$

Let $\Lambda \in \dot{H}^{2}$ be a vector divergence free and $\Xi \in L^{2}$ a scaler test functions. Taking the inner product and integrating over $[0, t]$, for $t \in\left[0, T^{*}\right]$, we obtain

$$
\begin{gathered}
\left\langle\theta_{k}(t), \Xi\right\rangle-\left\langle\theta_{k}(0), \Xi\right\rangle-\int_{0}^{t}\left\langle\theta_{k}, \Delta \Xi\right\rangle d \tau+\int_{0}^{t}\left\langle B\left(u_{k}, \theta_{k}\right), \Xi\right\rangle d \tau=0, \\
\left\langle v_{k}(t), \Lambda\right\rangle-\left\langle v_{k}(0), \Lambda\right\rangle-\int_{0}^{t}\left\langle v_{k}, \Delta \Lambda\right\rangle d \tau+\int_{0}^{t}\left\langle\tilde{B}\left(u_{k}, v_{k}\right), \Lambda\right\rangle d \tau-\int_{0}^{t}\left\langle\theta_{k} e_{3}, \Lambda\right\rangle d \tau=0 .
\end{gathered}
$$

To handle the nonlinear terms, we use a standard compactness argument (thanks to the uniform bounds obtained with respect to $\alpha_{k}$ above) so that $\tilde{B}\left(u_{k}, v_{k}\right) \rightarrow B(u, u)$ and $B\left(u_{k}, \theta_{k}\right) \rightarrow B(u, \theta)$. Hence, taking the limit, for every $t \in\left[0, T^{*}\right] \backslash E$, to obtain

$$
\langle\theta(t), \Xi\rangle-\langle\theta(0), \Xi\rangle-\int_{0}^{t}\langle\theta, \Delta \Xi\rangle d \tau+\int_{0}^{t}\langle B(u, \theta), \Xi\rangle d \tau=0,
$$




$$
\langle u(t), \Lambda\rangle-\langle u(0), \Lambda\rangle-\int_{0}^{t}\langle u, \Delta \Lambda\rangle d \tau+\int_{0}^{t}\langle B(u, u), \Lambda\rangle d \tau-\int_{0}^{t}\left\langle\theta e_{3}, \Lambda\right\rangle d \tau=0 .
$$

Especially, every strong solution fulfills the energy estimates (1.11), so one deduces the energy estimates (1.13) by taking the lower limit as $\alpha_{k} \rightarrow 0^{+}$.

Acknowledgement: The authors gratefully acknowledge the approval and the support of this research study by the grant number SCI-2018-3-9-F-8032 from the Deanship of Scientific Research at Northern Border University, Arar, K. S. A.

Conflicts of Interest: The author(s) declare that there are no conflicts of interest regarding the publication of this paper.

\section{REFERENCES}

[1] Y. Cao, E. M. Lunasin, E. S. Titi, Global well-posedness of the three-dimensional viscous and inviscid simplified Bardina turbulence models. Commun. Math. Sci. 4 (2006), 823-848.

[2] A. Chaabani, R. Nasfi, R. Selmi, M. Zaabi, Well-posedness and convergence results for strong solution to a 3D-regularized Boussinesq system, Math. Meth. Appl. Sci. (2016). https://doi.org/10.1002/mma.3950.

[3] Cheskidov, A., Holm, D., Olson, E., Titi, E.: On a Leray- $\alpha$ model of turbulence. Proc. R. Soc. Lond. Ser. A 461 (2005), 629-649.

[4] B. Cushman-Roisin, J. M. Beckers, Introduction to geophysical Fluid Dynamics, Series in International Geophysics. Academic Press, 2nd edition, 2011.

[5] C. Foias, D. D. Holm, E. S. Titi, The Navier-Stokes-alpha model of fluid turbulence. Physica D, 152 (2001), 505-519.

[6] D. D. Holm, C. Jeffery, S. Kurien, D. Livescu, M. A. Taylor, B. A. Wingate, The LANS- $\alpha$ model for computing turbulence. Los Alamos Sci. 29 (2005), 152-171.

[7] D. D. Holm, J. E. Marsden, T. S. Ratiu, The Euler-Poincaré equations and semidirect products with applications to continuum theories. Adv. Math. 137(1) (1998), 1-81.

[8] A. A. Ilyin, E. M. Lunasin, E. S. Titi, A modified Leray-alpha subgrid-scale model of turbulence. Nonlinearity, 19 (2006), $879-897$.

[9] J. Leray, Sur le mouvement d'un liquide visquex emplissant l'espace. Acta Math. 63 (1934), 193-248.

[10] J. S. Linshiz, E. S. Titi, Analytical study of certain magnetohydrodynamic- $\alpha$ models, J. Math. Phys. 48 (2007), 065504.

[11] J. Robinson, J. Rodrigo, W. Sadowski, The Three-Dimensional Navier-Stokes Equations: Classical Theory (Cambridge Studies in Advanced Mathematics), Cambridge University Press, Cambridge, 2016.

[12] R. Selmi, Global Well-Posedness and Convergence Results for the 3D-Regularized Boussinesq System. Canad. J. Math. 64(6) (2012), 1415-1435. 